\title{
Image quality evaluation of dual-layer spectral CT in comparison to single-layer CT in a reduced-dose setting
}

\author{
Thuy Duong Do ${ }^{1} \cdot$ Stephan Rheinheimer ${ }^{1}$ Hans-Ulrich Kauczor ${ }^{1,2} \cdot$ Wolfram Stiller $^{1,2} \cdot$ Tim Weber $^{1}$. \\ Stephan Skornitzke ${ }^{1}$
}

Received: 31 December 2019 / Revised: 6 March 2020 / Accepted: 14 April 2020 / Published online: 11 May 2020

(C) The Author(s) 2020

\begin{abstract}
Objectives To quantitatively and qualitatively evaluate image quality in dual-layer CT (DLCT) compared to single-layer CT (SLCT) in the thorax, abdomen, and pelvis in a reduced-dose setting.

Methods Intraindividual, retrospective comparisons were performed in 25 patients who received at least one acquisition of all three acquisition protocols $\mathrm{SLCT}_{\text {low }}(100 \mathrm{kVp}), \mathrm{DLCT}_{\text {high }}(120 \mathrm{kVp})$, and $\mathrm{DLCT}_{\text {low }}(120 \mathrm{kVp})$, all covering the venous-phase thorax, abdomen, and pelvis with matched $\mathrm{CTDI}_{\mathrm{vol}}$ between $\mathrm{SLCT}_{\mathrm{low}}$ and $\mathrm{DLCT}_{\text {low }}$. Reconstruction parameters were identical between all scans. Image quality was assessed quantitatively at 10 measurement locations in the thorax, abdomen, and pelvis by two independent observers, and subjectively with an intraindividual forced choice test between the three acquisitions. Doselength product (DLP) and $\mathrm{CTDI}_{\mathrm{vol}}$ were extracted for dose comparison.

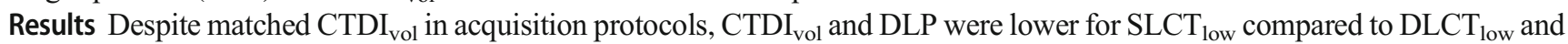
$\mathrm{DLCT}_{\text {high }}\left(\mathrm{DLP} 408.58,444.68,647.08 \mathrm{mGy} \cdot \mathrm{cm}\right.$, respectively; $p<0.0004$ ), as automated tube current modulation for DLCT $\mathrm{T}_{\text {low }}$ reached the lower limit in the thorax (mean $66.1 \mathrm{mAs}$ vs limit $65 \mathrm{mAs}$ ). Noise and CNR were comparable between $\mathrm{SLCT}_{\text {low }}$ and $\mathrm{DLCT}_{\text {low }}$ ( $p$ values, 0.29-0.51 and 0.05-0.20), but CT numbers were significantly higher for organs and vessels in the upper

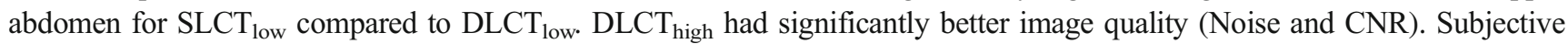
image quality was superior for $\mathrm{DLCT}_{\text {high }}$, but no difference was found between $\mathrm{SLCT}_{\text {low }}$ and $\mathrm{DLCT}_{\text {low }}$.

Conclusions $\mathrm{DLCT}_{\text {low }}$ showed comparable image quality to $\mathrm{SLCT}_{\text {low }}$, with the additional possibility of spectral post-processing. Further dose reduction seems possible by decreasing the lower limit of the tube current for the thorax.

Key Points

- Clinical use of reduced-dose DLCT is feasible despite the required higher tube potential.

- DLCT with reduced dose shows comparable objective and subjective image quality to reduced-dose SLCT.

- Further dose reduction in the thorax might be possible by adjusting mAs thresholds.
\end{abstract}

Keywords Tomography, X-ray computed $\cdot$ Radiation exposure $\cdot$ Thorax $\cdot$ Abdomen

\begin{tabular}{|c|c|c|c|}
\hline \multicolumn{2}{|c|}{ Abbreviations } & CT & Computed tomography \\
\hline \multirow{2}{*}{ AP } & Anterior-posterior & $\mathrm{CTDI}_{\mathrm{vol}}$ & Volumetric computed tomography dose index \\
\hline & Controct to noicon & DLCT & Dual-layer spectral CT \\
\hline \multirow{3}{*}{$\triangle$} & \multirow{3}{*}{$\begin{array}{l}\text { Stephan Skornitzke } \\
\text { stephan.skornitzke@med.uni-heidelberg.de }\end{array}$} & DRI & Dose right index \\
\hline & & DRL & Diagnostic reference levels \\
\hline & & ICC & Intraclass-correlation coefficient \\
\hline \multirow{3}{*}{ (1) } & \multirow{3}{*}{$\begin{array}{l}\text { Clinic for Diagnostic and Interventional Radiology (DIR), } \\
\text { Heidelberg University Hospital, Im Neuenheimer Feld 110, } \\
69120 \text { Heidelberg, Germany }\end{array}$} & ROI & Region of interest \\
\hline & & SD & Standard deviation \\
\hline & & SLCT & Single-layer CT \\
\hline \multirow[t]{2}{*}{2} & Translational Lung Research Center (TLRC), Member of the German & SSDE & Size-specific dose estimate \\
\hline & Center for Lung Research (DZL), Heidelberg, Germany & VMSI & Virtual monochromatic spectral imaging \\
\hline
\end{tabular}




\section{Introduction}

The introduction of dual-layer detector technology in computed tomography (CT) enabled the acquisition of spectral data for all performed scans without the need of an additional CT $\mathrm{x}$-ray tube or additional acquisitions. Dual-layer spectral CT (DLCT) acquisitions allow material decomposition (virtual non-contrast, iodine-only imaging, and effective atomic numbers) as well as the calculation of virtual monoenergetic images. Several clinical studies have already been performed showing the advantages of DLCT for head CT for imaging intracerebral lesions and hemorrhage, for thoracic $\mathrm{CT}$, for vertebral CT for differentiating bone lesions, and for abdominal CT angiographies for improved delineation of visceral arteries [1-5]. However, for the image acquisition of such data, a tube potential of either $140 \mathrm{kVp}$ or $120 \mathrm{kVp}$ is necessary to allow for spectral decomposition under the exploitation of the energy-specific x-ray absorption of different materials. In contrast to changes in tube current, changes in tube potential have a non-linear effect on radiation dose: in comparison to $80 \mathrm{kVp}$, the $\mathrm{x}$-ray tube output (i.e., air kerma or exposure) is 1.5 times higher for $100 \mathrm{kVp}, 2.5$ times higher for $120 \mathrm{kVp}$, and 3.4 times higher for $140 \mathrm{kVp}$ [6].

While patient radiation exposure has long been a topic of interest in CT, as CT accounts for 49-66\% of overall patient radiation exposure, this interest has recently led to new regulations in the European Union via the EURATOM directive, with the deadline for implementation into federal law in 2018 $[7,8]$. Based on surveys, diagnostic reference levels (DRLs) at the 75th percentile of dose distribution have been defined as the limits of appropriate practice for CT acquisitions. In our institution, acquisition protocols for the thorax, abdomen, and pelvis have been previously optimized to reduce patient radiation exposure beyond these legal requirements. This was achieved, in part, by reducing tube potential from 120 to $100 \mathrm{kVp}$ for single-layer CT (SLCT), resulting in an average patient dose $60 \%$ below the federal DRL for the thorax, abdomen, and pelvis, which is a DLP of $1000 \mathrm{mGy} \cdot \mathrm{cm}$ and a $\mathrm{CTDI}_{\mathrm{vol}}$ of $13 \mathrm{mGy}$.

With the installation of a novel DLCT, vendor's recommended settings were applied including a tube potential of $120 \mathrm{kVp}$ (acquisition protocol $\mathrm{DLCT}_{\text {high }}$ ), which led to an increase in radiation exposure in comparison to previous reduced-dose SLCT protocols (acquisition protocol $\mathrm{SLCT}_{\text {low }}$ ), albeit still remaining below the applicable diagnostic reference levels. After initial experience with $\mathrm{DLCT}_{\text {high }}$, the tube current was reduced (acquisition protocol $\mathrm{DLCT}_{\text {low }}$ ) to match the $\mathrm{CTDI}_{\mathrm{vol}}$ of the reduced-dose protocol previously performed on the SLCT scanner at $100 \mathrm{kVp}\left(\mathrm{SLCT}_{\text {low }}\right)$.

The aim of the study was to quantitatively and qualitatively compare the image quality and radiation exposure of the SLCT acquisition protocol with reduced dose, $\mathrm{SLCT}_{\text {low }}$ (41\% of the national DRL), and the DLCT acquisition protocol with reduced dose, $\mathrm{DLCT}_{\text {low }}$ (44\% of the DRL). Thus, by comparing DLCT $_{\text {low }}$ and SLCT $_{\text {low }}$, we want to aid in the deployment of protocols with reduced dose in DLCT with image quality comparable to previous low-tube potential $\mathrm{SLCT}_{\text {low }}$, while allowing to benefit from the spectral information in a clinical setting.

\section{Material and methods}

\section{Ethics approval and consent}

This retrospective study was approved by the institutional review board. The need for written informed consent was waived.

\section{Patient selection}

Ninety-six patients who underwent low-dose DLCT $\left(\mathrm{DLCT}_{\text {low }}\right)$ and previous low-dose $\mathrm{SLCT}_{\text {low }}$ with clinical indication for oncological staging, since October 2018, were screened for inclusion into the study (Fig. 1). Intensive care patients and obese patients were excluded from the study, as low-dose protocols are not routinely used in these circumstances and obese patients require adapted acquisition protocols to guarantee adequate image quality. Arterial-phase acquisitions or venous-phase examinations with additional arterial angiography phase were not considered for the study to ensure comparability of the evaluated acquisitions, as those are to prone to effects of contrast agent volume, injection speed of the contrast agent, and circulation and cardiac output. In consequence, only patients with a routine $\mathrm{CT}$ acquisition of the thorax, abdomen, and pelvis in the venous phase were included into the study. All patients received a similar total amount of contrast agent in all their examinations with a maximum intraindividual difference of $5 \mathrm{ml}$. Patients with diffuse liver parenchyma diseases (liver cirrhosis and liver metastases), liver perfusion deficit, or intracorporal metal implants (e.g., hip prosthesis or spinal hardware) were excluded from the study. Furthermore, non-standard CT examinations as defined by differences in the acquisition protocol were excluded from the study. In summary, 31 patients were included into the study, who previously underwent $51 \mathrm{SLCT}_{\text {low }}$ examinations, $39 \mathrm{DLCT}_{\text {high }}$ examinations, and $34 \mathrm{DLCT}_{\text {low }}$ examinations with adjusted parameters (Fig. 1). The mean interval between the acquisitions with the different acquisition protocols was $224.6 \pm 89.8$ days (median, 190 days) between $\mathrm{SLCT}_{\text {low }}$ and $\mathrm{DLCT}_{\text {high }}$ and $182.2 \pm 70.5$ days (median, 183.5 days) be-

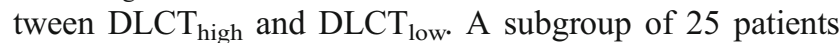
had been examined with all three CT acquisition protocols and was used for qualitative analysis. 
Fig. 1 Flowchart illustrating study inclusion and exclusion criteria. In total, 31 patients could be included into the study with repeated CT scans consisting of $51 \mathrm{SLCT}_{\text {low }}, 39 \mathrm{DLCT}_{\text {high }}$, and 34 $\mathrm{DLCT}_{\text {low }}$

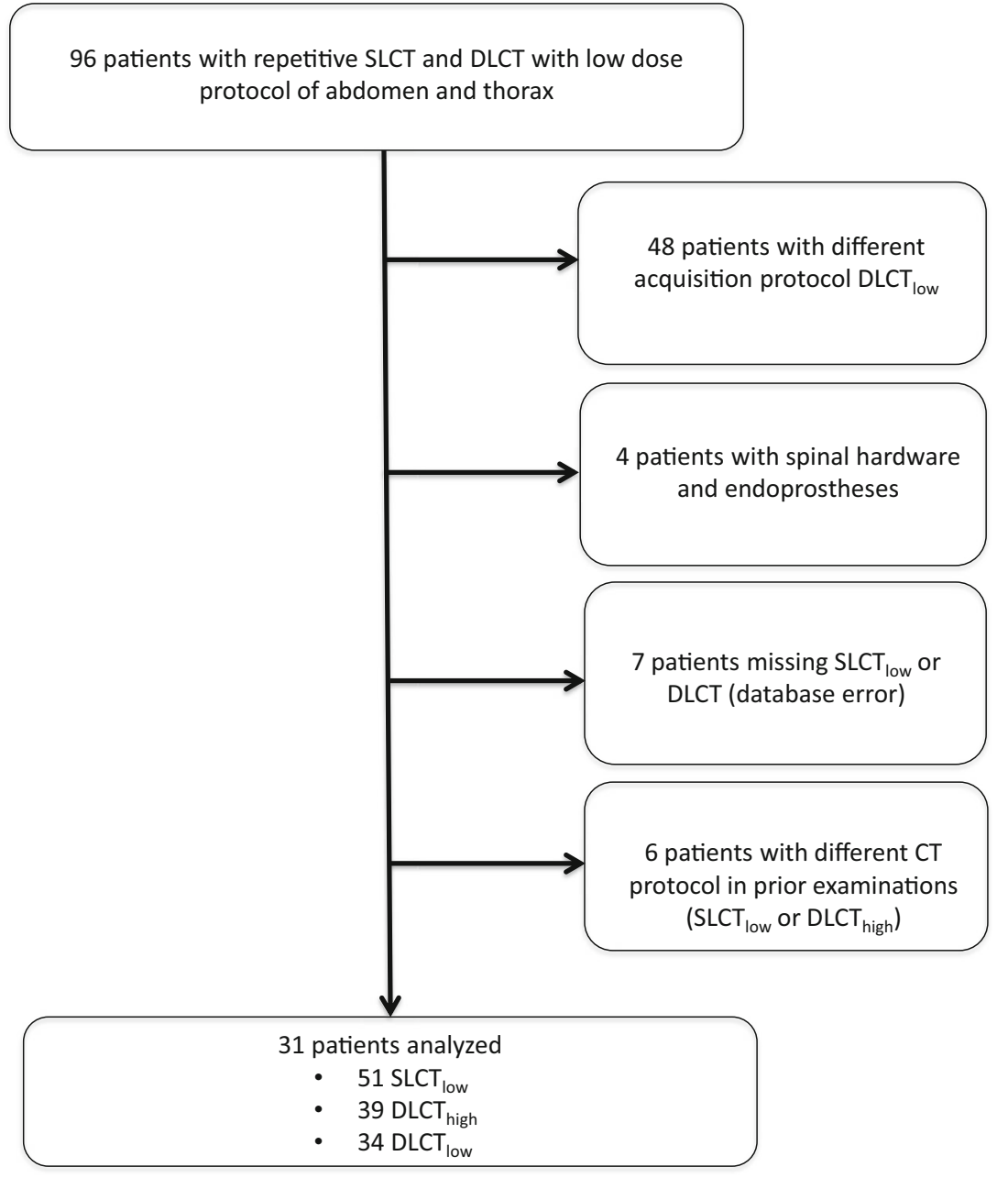

\section{CT acquisition parameters}

CT acquisitions were performed with a standard acquisition protocol for oncological staging covering the thorax, abdomen, and pelvis by a single continuous acquisition (all three scan protocols). Acquisition parameters of $\mathrm{SLCT}_{\text {low }}$ (iCT, Philips Healthcare), $\mathrm{DLCT}_{\text {low }}$, and $\mathrm{DLCT}_{\text {high }}$ (IQon Spectral CT, Philips Healthcare) are shown in Table 1. SLCT $\mathrm{S}_{\text {low }}$ was performed with a tube potential of $100 \mathrm{kVp}$, while all DLCTs were performed with a tube potential $120 \mathrm{kVp}$. For $\mathrm{DLCT}_{\text {high, }}$, standard acquisition settings were used as per the manufacturer's presets. For $\mathrm{DLCT}_{\text {low, }}$, the automated tube current modulation (DoseRight 3D-DOM, Philips Healthcare) was adjusted by reducing dose right index (DRI) from 17 to 13 to match the $\mathrm{SLCT}_{\text {low }}$ protocol, achieving a similar predicted CTDI ${ }_{\mathrm{vol}}$, as displayed when editing the acquisition protocol in the CT scanner interface $\left(\mathrm{DLCT}_{\text {low }} 7.5 \mathrm{mGy}, \mathrm{SLCT}_{\text {low }} 7.8 \mathrm{mGy}\right.$ ). Accordingly, predicted average mAs was lower for DLCTl $\mathrm{ow}_{\text {w }}$ (74 mAs) compared to DLCT $_{\text {high }}(116 \mathrm{mAs})$.

Collimation (single collimation width $0.625 \mathrm{~mm}$ and total collimation width $40 \mathrm{~mm}$ ) and pitch $(0.798)$ was identical for all scans.
Table 1 CT acquisition parameters for $\mathrm{SLCT}_{\text {low }}$, $\mathrm{DLCT}_{\text {low }}$, and $\mathrm{DLCT}_{\text {high }}$

\begin{tabular}{lccc}
\hline & SLCT $_{\text {low }}$ & DLCT $_{\text {low }}$ & DLCT $_{\text {high }}$ \\
\hline Tube potential & $100 \mathrm{kVp}$ & $120 \mathrm{kVp}$ & $120 \mathrm{kVp}$ \\
$\begin{array}{l}\text { Automated tube current modulation } \\
\quad(\text { dose right index) }\end{array}$ & 14 & 13 & 17 \\
Predicted CTDI & & $7.5 \mathrm{mGy}$ & $11.5 \mathrm{mGy}$ \\
Predicted average mAs & $7.8 \mathrm{mGy}$ & $74 \mathrm{mAs}$ & $116 \mathrm{mAs}$ \\
Minimum mAs & $174 \mathrm{mAs}$ & $65 \mathrm{mAs}$ & $65 \mathrm{mAs}$ \\
Maximum mAs & $65 \mathrm{mAs}$ & $300 \mathrm{mAs}$ & $350 \mathrm{mAs}$ \\
\hline
\end{tabular}


All examinations were conducted in craniocaudal direction and supine position, with automatic exposure control as described above. For all acquisitions, iohexol contrast agent (AccupaqueTM 350, GE Healthcare) was used. Contrast agent application was performed using a power injector with an injection rate of $3 \mathrm{ml} / \mathrm{s}$. A routine biphasic contrast-injection scan protocol was used covering the thorax, abdomen, and pelvis in the venous phase. The first contrast agent injection and saline solution chaser bolus was followed by a 30 -s break and a second contrast agent injection with a saline solution chaser bolus. Then the CT acquisition was performed $60 \mathrm{~s}$ after the second contrast agent injection. The combined acquisition of thorax and abdominal region in the venous phase achieves better delineation of the lymph nodes, thoracic wall, and mediastinal soft tissue with less artifacts than a thoracic arterial phase for oncological staging [9]. As previously stated, only patients who received a venous-phase CT acquisition of the thorax, abdomen, and pelvis were included in the study.

\section{Image reconstruction and post-processing}

For all examinations, axial series with a slice thickness of $3 \mathrm{~mm}$ and increment of $1.5 \mathrm{~mm}$ were reconstructed. Images were reconstructed with a vendor-specific iterative reconstruction algorithm, IMR at level 1 (Iterative Model Reconstruction, Philips Healthcare), soft-tissue setting, and a standard abdomen setting (window center/width 40/400 HU).

\section{Dose and image analysis}

Quantitative and qualitative analysis were performed by two radiologists with 7 and 6 years of experience in abdominal radiology. For quantitative analysis, all acquisitions were included. For qualitative analysis, the most recent examination of each acquisition protocol SLCT $_{\text {low }}, \mathrm{DLCT}_{\text {high }}$, and $\mathrm{DLCT}_{\text {low }}$ ) was included and only patients that were scanned with all three acquisition protocols were included.

Patients' anterior-posterior and lateral diameters were measured separately for the thorax at the level of tracheal bifurcation, the upper abdomen at the level of the portal vein, and the lower abdomen at the level of lumbar spine L4 (Fig. 2). Total scan lengths were calculated from the images.

Quantitative image analysis was performed by placement of regions of interest (ROIs) in axial slices in the following positions (Fig. 2):

a) Level of the tracheal bifurcation: in the subcutaneous fat, peripheral lung parenchyma, and air within the trachea

b) Level of the portal vein: in the subcutaneous fat of the right upper abdomen, liver parenchyma, portal vein, aorta, and spleen
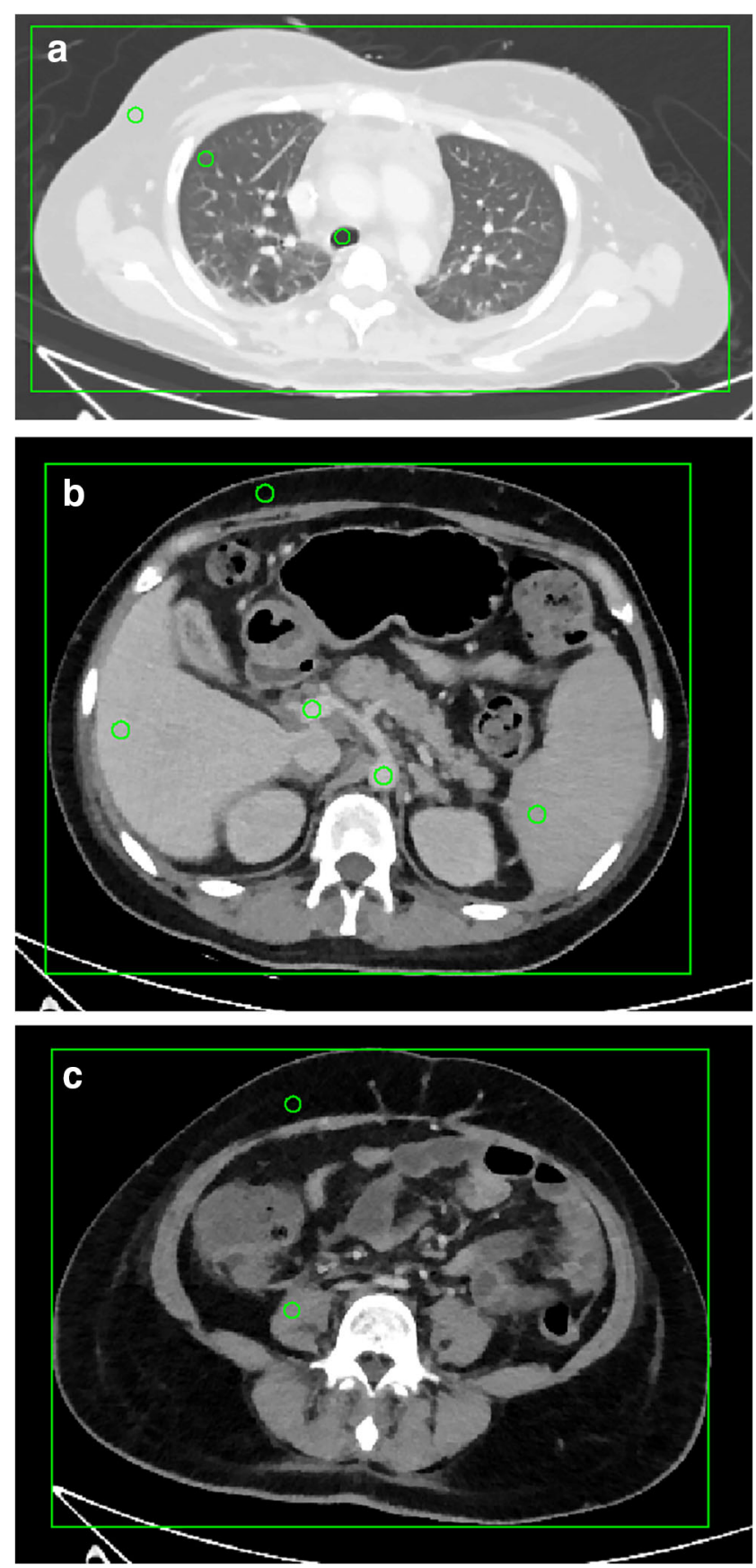

Fig. 2 Image examples of regions of interest (ROIs) used for the quantitative evaluation in the thorax at the height of the tracheal bifurcation (a), the upper abdomen at the height of the portal vein (b), and the lower abdomen at the level of the lumbar spine L4 (c). Rectangle for measurements of the lateral and anterior-posterior diameter of the torso

c) Level of the lumbar spine L4: in the subcutaneous fat of the right lower abdomen and psoas muscle

For all three axial slices, the slice-specific tube currentexposure time product, lateral, and anterior-posterior torso diameter were determined. An in-house developed software was used for semiautomatic quantitative image quality 
analysis to maintain constant ROI area size of $50 \mathrm{~mm}^{2}$ in order to enhance reproducibility and time-efficient evaluation [10]. Noise was defined as the standard deviation (SD) of CT numbers of the subcutaneous fat on the right side of the ventral thorax or abdomen for improved comparability. Mean and SD of CT numbers were recorded and CNR was calculated as follows:

\section{(I) $\mathrm{CNR}=\left(\mathrm{Mean}_{\mathrm{ORGAN}}-\mathrm{Mean}_{\mathrm{FAT}}\right) / \mathrm{SD}_{\mathrm{FAT}}$}

The following ROIs were used to calculate CNR in comparison to the subcutaneous fat on their respective axial slice: lung parenchyma (thorax), liver parenchyma (upper abdomen), and psoas muscle (lower abdomen).

For qualitative image evaluation comparing $\mathrm{SLCT}_{\text {low }}$, $\mathrm{DLCT}_{\text {high }}$, and $\mathrm{DLCT}_{\text {low }}$ (Fig. 3), a forced choice method was used for a blinded and randomized side-by-side review. For randomization, both the order of patients and evaluated series were randomized by the statistician, who was not involved in qualitative image evaluation, using the random function of
EXCEL (Microsoft). Two observers independently performed the qualitative analysis on conventional $\mathrm{CT}$ images reconstructed from all three acquisition protocols. First, images from all three acquisition protocols were separately ranked for three body regions covered by the acquisition protocols taking into account the following criteria [11]:

- Thorax: noise in the subcutaneous fat of the right thorax, delineation of trachea, bronchi, and spinal cord

- Upper abdomen: noise in the right-sided subcutaneous fat, delineation of portal vein, aorta, and spinal cord

- Lower abdomen: noise in the right-sided subcutaneous fat, delineation of gluteal muscles, and spinal cord

Based on the rankings for the three body regions, unique ranks from 1 to 3 were allocated to the evaluated image series (i.e., observes were forced to choose which image has the best image quality compared to the others) as a total score, whereby a rank of 1 corresponded to the best image quality.

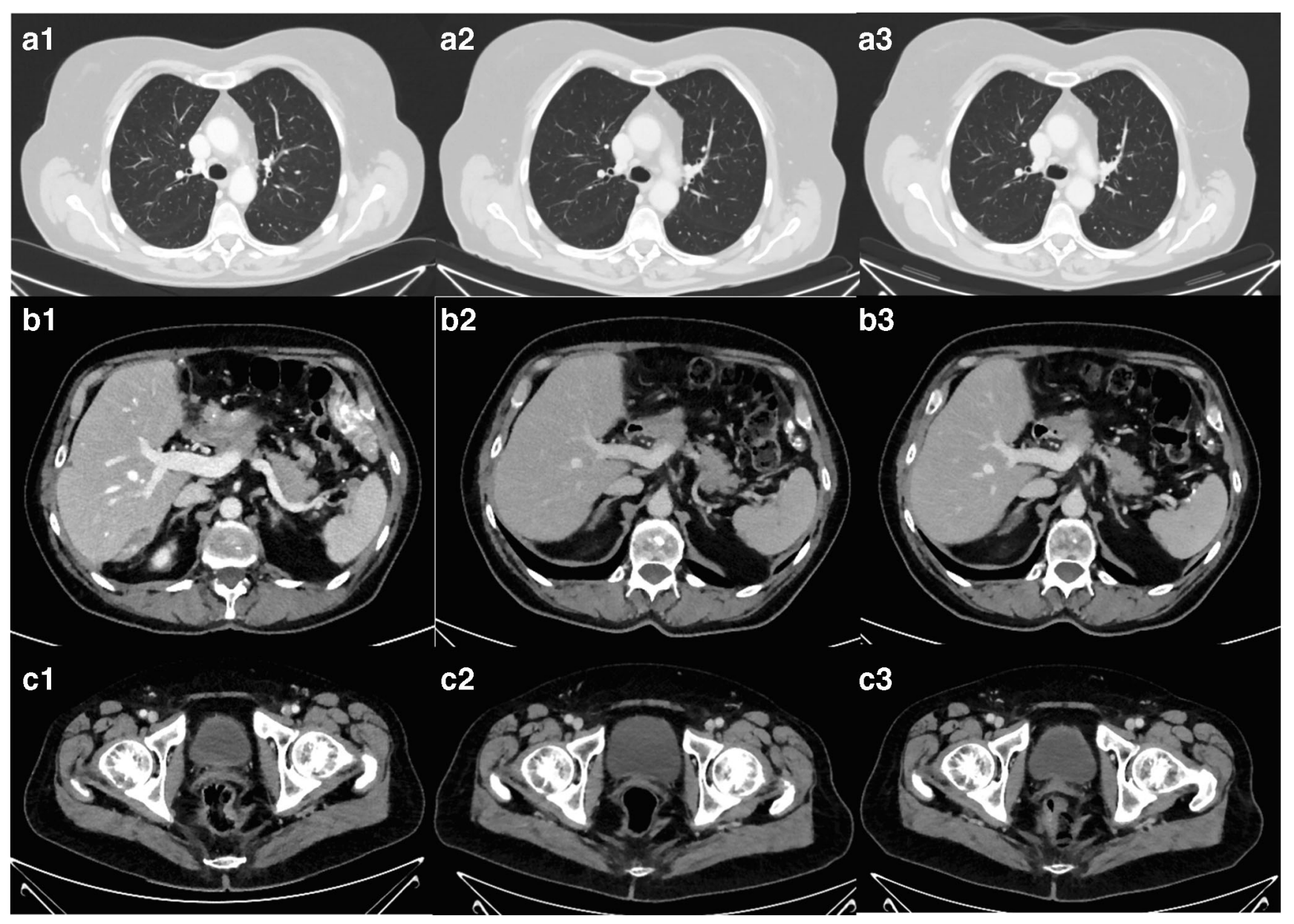

Fig. 3 Image examples from the same patient from conventional reduced-dose $\mathrm{SLCT}_{\text {low }}$ (column 1), $\mathrm{DLCT}_{\text {low }}$ (column 2), and $\mathrm{DLCT}_{\text {high }}$ (column 3 ) from thorax (a), upper abdomen (b), and pelvis (c). $\mathrm{DLCT}_{\text {high }}$ (column 2) showed best quantitative and qualitative analysis whereas $\mathrm{SLCT}_{\text {low }}$ (column 1) and $\mathrm{DLCT}_{\text {low }}$ (column 3) showed comparable results 
DLPs, $\mathrm{CTDI}_{\mathrm{vol}}$, and overall tube current-time product were extracted from the patient's dose report images sent to the picture archiving system. Size-specific dose estimates (SSDEs) for each patient and each body region were calculated according to the recommendations of the American Association of Physicsts in Medicine Task Group 204 [12]. $\mathrm{CTDI}_{\mathrm{vol}}$ and DLP are frequently used parameters for estimation of a patient's radiation exposure. However, SSDE is a more precise method for the patient's absorbed dose as it takes the patient's size into account [13]. To calculate SSDE for the individual anatomical region, a region-specific $\mathrm{CTDI}_{\mathrm{vol}, \text { region }}$ was estimated by dividing the $\mathrm{CTDI}_{\mathrm{vol}}$ for the complete acquisition by the average tube current-time product and multiplying it with the tube current-time product of the representative axial slice of each region (Fig. 2):

$$
\begin{aligned}
& \text { (II) } \mathrm{CTDI}_{\mathrm{vol}, \text { region }} \\
& =\mathrm{CTDI}_{\mathrm{vol}} \cdot \text { average } \mathrm{mAs}_{\text {total }} / \text { average } \mathrm{mAs}_{\text {region }}
\end{aligned}
$$

The resulting estimated region-specific $\mathrm{CTDI}_{\mathrm{vol}, \text { region }}$ was multiplied with the recommended conversion coefficient depending on the lateral and AP diameter of the selected anatomical region [13].

\section{Statistical analysis}

Statistical analysis was performed using SAS Version 9.4 (SAS Institute Inc.) and SPSS Version 19.0 (IBM). Descriptive statistics were calculated, determining means and standard deviations for normal distributed data. CT numbers, image noise approximated by the standard deviation of measured CT numbers, and CNR were analyzed for differences depending on the CT scanner and the acquisition protocol using a mixed model for unbalanced analysis of variances for repeated measures.

Qualitative ratings of image quality were analyzed for differences depending on the CT scanner and the acquisition protocol using non-parametric tests (Friedman test stratified for patients and Wilcoxon signed-rank test in a multistage design).

For the evaluation of the inter-rater agreement of the quantitative analysis, the intraclass correlation coefficient (ICC) was used and for the qualitative analysis Cohen's kappa was calculated and classified according to Landis and Koch [14]. The significance level for statistical testing was set at $p<0.05$.

\section{Results}

\section{Quantitative analysis}

Although the average scan length of DLCT $_{\text {low }}$ was significantly shorter than that for $\operatorname{SLCT}_{\text {low }}(p>0.0001)$ with an average difference of $13.8 \mathrm{~mm}$, DLP for DLCT $_{\text {low }}$ was significantly higher due to higher $\mathrm{CTDI}_{\mathrm{vol}}$ of $\mathrm{DLCT}_{\text {low }}$ in comparison to $\mathrm{SLCT}_{\text {low }}(444.68 \mathrm{mGy} \cdot \mathrm{cm}$ and $408.58 \mathrm{mGy} \cdot \mathrm{cm}$, respectively; $p=0.0004$ ) (Table 2). $\mathrm{CTDI}_{\mathrm{vol}}$ of $\mathrm{DLCT}_{\text {low }}(6.57 \mathrm{mGy})$ was $30 \%$ lower than that of $\mathrm{DLCT}_{\text {high }}(9.34 \mathrm{mGy} ; p<0.0001)$. Despite the efforts to match radiation dose, average $\mathrm{CTDI}_{\mathrm{vol}}$ was $14.4 \%$ higher for $\mathrm{DLCT}_{\text {low }}$ compared to $\mathrm{SLCT}_{\text {low }}$ $(p<0.0001)$. Similar to $\mathrm{CTDI}_{\mathrm{vol}}$ and DLP, the SSDE was lowest for $\mathrm{SLCT}_{\text {low }}$ while SSDE of $\mathrm{DLCT}_{\text {low }}$ was significantly less than $\mathrm{DLCT}_{\text {high }}$ for all body regions $(p<0.0001)$. $\mathrm{DLCT}_{\text {high }}$ had significantly higher CTDI $\mathrm{vol}(p<0.0001)$ and higher DLP $(p<0.0001)$ resulting from higher tube potential in comparison to $\mathrm{SLCT}_{\text {low }}$, despite comparable scan length to $\operatorname{SLCT}_{\text {low }}(p=0.56)$.

A decrease of the mean lateral diameter of patients over time was observed, with a maximum difference/change of $10.3 \mathrm{~mm}$ (Table 3 ). There was also a statistically significant difference of both the lateral thoracic and lower abdominal diameter between the first (and earliest) examination with SLCT $_{\text {low }}$ in comparison to the acquisitions with DLCT $_{\text {high }}$ ( $p<0.0006$ and 0.0029 , respectively), but the mean difference was only $5.6 \mathrm{~mm}$ (thorax) and $9.2 \mathrm{~mm}$ (lower abdomen). No significant differences in anterior-posterior (AP) diameter were observed for all three abdominal regions.

In general, the lowest tube current-time product was found for $\mathrm{DLCT}_{\text {low }}$ in all three regions, showing a statistically significant difference compared to $\mathrm{DLCT}_{\text {high }}$ in the lower and upper abdomen, but not in the thorax (Table 3). It has to be noted that for the thorax, the average tube current-time product of $\mathrm{DLCT}_{\text {low }}$ was $66.1 \mathrm{mAs}$, which is close to the minimum $\mathrm{mAs}$ of the tube current modulation preset at $65 \mathrm{mAs}$ (Fig. 4). Also, mAs for the upper and lower abdomen was lowest for $\mathrm{DLCT}_{\text {low, }}$ at approximately $49-51 \%$ of $\mathrm{SLCT}_{\text {low }}$.

Table 2 Evaluation of patient radiation exposure: means of scan length, CTDI $_{\mathrm{vol}}$, DLP, and SSDE of SLCT $\mathrm{low}_{\text {, }}, \mathrm{DLCT}_{\text {high }}$, and DLCT $\mathrm{DLw}_{\text {low }}$

\begin{tabular}{lllllrr}
\hline & Scan length (mm) & $\mathrm{CTDI}_{\text {vol }}(\mathrm{mGy})$ & DLP (mGy·cm) & SSDE Thorax (mGy) & $\begin{array}{l}\text { SSDE upper } \\
\text { abdomen (mGy) }\end{array}$ & $\begin{array}{l}\text { SSDE lower } \\
\text { abdomen (mGy) }\end{array}$ \\
\hline $\mathrm{SLCT}_{\text {low }}$ & $640.71 \pm 39.52$ & $5.74 \pm 1.26$ & $408.58 \pm 107.58$ & $5.00 \pm 1.20$ & $8.41 \pm 1.79$ & $7.28 \pm 1.72$ \\
$\mathrm{DLCT}_{\text {low }}$ & $626.91 \pm 38.77$ & $6.57 \pm 0.72$ & $444.68 \pm 57.63$ & $7.28 \pm 0.66$ & $9.19 \pm 1.04$ & $8.30 \pm 0.66$ \\
$\mathrm{DLCT}_{\text {high }}$ & $642.04 \pm 37.18$ & $9.34 \pm 2.13$ & $647.08 \pm 155.12$ & $7.92 \pm 0.91$ & $14.11 \pm 2.62$ & $11.52 \pm 1.96$ \\
\hline
\end{tabular}


Table 3 Evaluation of automatic exposure control: means and standard deviations of patients' lateral and anterior-posterior (AP) diameter, applied tube current-time product at the measurement location, and results of statistical testing. Statistical significance of results is indicated by italics

\begin{tabular}{|c|c|c|c|c|c|c|}
\hline \multirow[t]{2}{*}{ Anatomic location } & \multirow[t]{2}{*}{$\operatorname{SLCT}_{\text {low }}(*)$} & \multirow[t]{2}{*}{$\operatorname{DLCT}_{\text {low }}(* *)$} & \multirow[t]{2}{*}{$\operatorname{DLCT}_{\text {high }}(* * *)$} & \multicolumn{3}{|l|}{$p$ value } \\
\hline & & & & $*$ vs. $* *$ & $*$ vs. $* * *$ & $* *$ vs. $* * *$ \\
\hline \multicolumn{7}{|l|}{ Thorax } \\
\hline Lateral (mm) & $389.9 \pm 31.3$ & $379.6 \pm 31.7$ & $384.3 \pm 31.6$ & 0.0054 & 0.0006 & 0.53 \\
\hline $\mathrm{AP}(\mathrm{mm})$ & $231.3 \pm 31.3$ & $229.6 \pm 28.5$ & $237.5 \pm 31.0$ & 0.70 & 0.74 & 0.51 \\
\hline mAs & $98.8 \pm 29.1$ & $66.1 \pm 3.0$ & $74.3 \pm 12.6$ & $<0.0001$ & $<0.0001$ & 0.11 \\
\hline \multicolumn{7}{|l|}{ Upper abdomen } \\
\hline Lateral (mm) & $326.5 \pm 23.6$ & $320.4 \pm 25.9$ & $326.3 \pm 27.1$ & 0.027 & 0.86 & 0.052 \\
\hline $\mathrm{AP}(\mathrm{mm})$ & $247.2 \pm 29.8$ & $242.1 \pm 32.0$ & $252.7 \pm 35.9$ & 0.56 & 0.20 & 0.087 \\
\hline mAs & $151.9 \pm 41.5$ & $77.4 \pm 14.4$ & $123.9 \pm 35.4$ & $<0.0001$ & $<0.0001$ & $<0.0001$ \\
\hline \multicolumn{7}{|l|}{ Lower abdomen } \\
\hline Lateral (mm) & $348.5 \pm 36.9$ & $339.3 \pm 36.0$ & $342.7 \pm 39.5$ & 0.0055 & 0.0029 & 0.88 \\
\hline $\mathrm{AP}(\mathrm{mm})$ & $236.6 \pm 31.3$ & $233.7 \pm 34.4$ & $240.9 \pm 35.4$ & 0.68 & 0.46 & 0.77 \\
\hline $\mathrm{mAs}$ & $174.1 \pm 58.5$ & $80.7 \pm 25.4$ & $116.9 \pm 34.4$ & $<0.001$ & $<0.0001$ & $<0.0001$ \\
\hline
\end{tabular}

There was no significant difference in noise between $\mathrm{SLCT}_{\text {low }}, \mathrm{DLCT}_{\text {low }}$, and DLCT $\mathrm{Digh}_{\text {hi }}$ for the thorax and upper abdomen ( $p$ between 0.08 and 0.61 ). No significant difference in noise was found between $\mathrm{SLCT}_{\text {low }}$ and $\mathrm{DLCT}_{\text {low }}$ in the lower abdomen $(p=0.51)$. In general, noise was lowest for $\mathrm{DLCT}_{\text {high }}$, but the difference was only statistically significant in the lower abdomen $(p<0.05)$ (Table 4). No significant difference was found for CNR in the thorax and upper abdomen between $\mathrm{SLCT}_{\text {low }}$ and $\mathrm{DLCT}_{\text {low }}$. In the lower abdomen, CNR for DLCT $_{\text {low }}$ was significantly lower than that for $\mathrm{DLCT}_{\text {high, }}$, but no significant difference was found between $\mathrm{DLCT}_{\text {low }}$ and $\mathrm{SLCT}_{\text {low }}$. As for CT numbers, highest values were observed for vessels, e.g. aorta and portal vein, in comparison to organ parenchyma due to the use of contrast agent (Table 5). CT numbers of vessels in $\mathrm{SLCT}_{\text {low }}$ were significantly higher than those for both $\mathrm{DLCT}_{\text {low }}$ and
DLCT $_{\text {high }}$ which can be attributed to higher CT numbers observed for contrast agents at the lower tube potential of $100 \mathrm{kVp}$ versus $120 \mathrm{kVp}$. CT numbers for $\mathrm{DLCT}_{\text {high }}$ and DLCT $_{\text {low }}$ in organ parenchyma did not show significant differences, except for the liver, which can show considerable contrast agent uptake, where the mean difference was 13.7 HU between SLCT $_{\text {low }}$ and DLCT high. $_{\text {. }}$

\section{Qualitative analysis}

No significant image quality differences were observed between $\mathrm{SLCT}_{\text {low }}$ and $\mathrm{DLCT}_{\text {low }}(p=0.97)$. Overall, best image quality was observed for $\mathrm{DLCT}_{\text {high }}$ (mean rank $\pm \mathrm{SD}, 1.62 \pm$ $0.62)$ in comparison to $\operatorname{SLCT}_{\text {low }}(2.2 \pm 0.71)$ and $\mathrm{DLCT}_{\text {low }}$ $(2.18 \pm 0.72)$ with $p<0.01$ (Fig. 5).
Fig. 4 Example of tube currenttime product curves for the three evaluated acquisition protocols in one patient. Note the changes in tube current in the different anatomical regions caused by the automated exposure control, as well as the changes during the rotation of the x-ray tube around the patient table

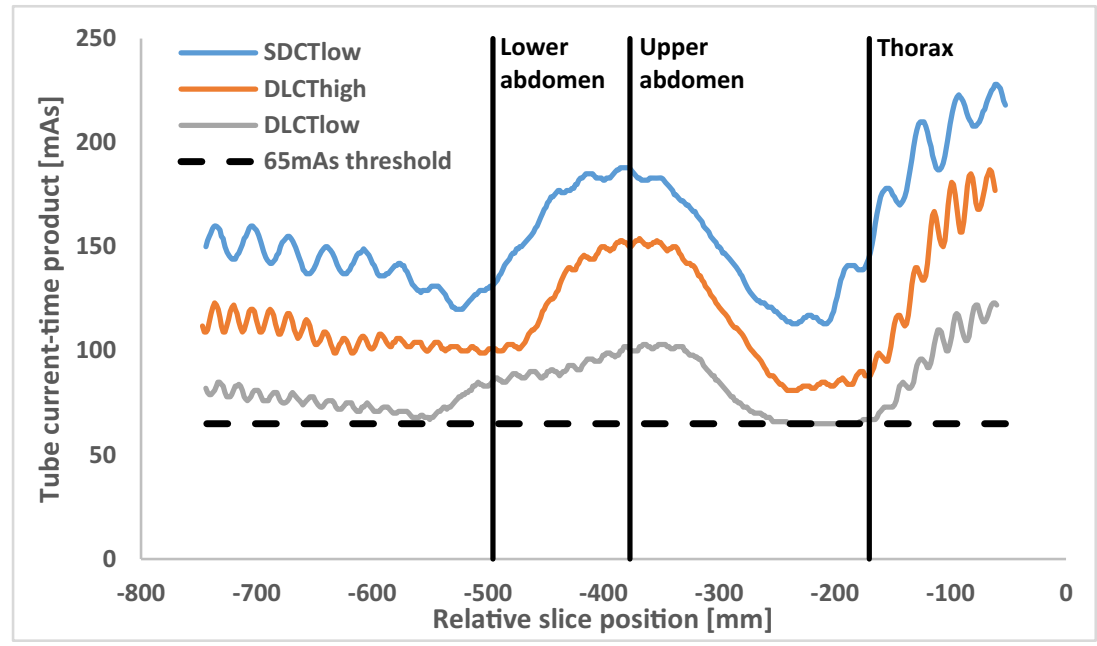


Table 4 Results of the quantitative image quality evaluation: image noise and CNR. Statistical significance of results is indicated by italics

\begin{tabular}{|c|c|c|c|c|c|c|}
\hline \multirow{2}{*}{$\begin{array}{l}\text { Quantitative parameters } \\
\text { Anatomic location }\end{array}$} & \multirow[t]{2}{*}{$\operatorname{SLCT}_{\text {low }}(*)$} & \multirow[t]{2}{*}{$\operatorname{DLCT}_{\text {low }}(* *)$} & \multirow[t]{2}{*}{$\operatorname{DLCT}_{\text {high }}(* * *)$} & \multicolumn{3}{|l|}{$p$ value } \\
\hline & & & & $*$ vs. $* *$ & $*$ vs. $* * *$ & $* *$ vs. $* * *$ \\
\hline \multicolumn{7}{|l|}{ Thorax } \\
\hline Noise (fat) (HU) & $5.4 \pm 2.0$ & $5.2 \pm 2.6$ & $5.0 \pm 2.2$ & 0.29 & 0.08 & 0.53 \\
\hline CNR (lung) & $-157.8 \pm 52.7$ & $-165.4 \pm 45.6$ & $-173.9 \pm 51.5$ & 0.20 & 0.020 & 0.33 \\
\hline \multicolumn{7}{|l|}{ Upper abdomen } \\
\hline Noise (fat) (HU) & $5.9 \pm 1.6$ & $6.1 \pm 2.2$ & $5.6 \pm 1.6$ & 0.32 & 0.61 & 0.16 \\
\hline CNR (liver) & $41.7 \pm 12.7$ & $38.6 \pm 12.3$ & $39.8 \pm 11.8$ & 0.05 & 0.10 & 0.71 \\
\hline \multicolumn{7}{|l|}{ Lower abdomen } \\
\hline Noise (fat) (HU) & $5.1 \pm 1.5$ & $5.1 \pm 1.2$ & $4.6 \pm 1.1$ & 0.51 & 0.0018 & 0.02 \\
\hline CNR (psoas) & $37.3 \pm 10.8$ & $35.0 \pm 8.6$ & $39.5 \pm 10.4$ & 0.18 & 0.12 & 0.0085 \\
\hline
\end{tabular}

ICC for quantitative inter-rater agreement was on average 0.89. Interobserver agreement for qualitative analysis was moderate with Cohen's kappa of 0.42 according to the classification of Landis and Koch [14].

\section{Discussion}

The necessity to increase the tube potential to at least $120 \mathrm{kVp}$ for spectral imaging with current DLCT results in an increase of patient radiation exposure, compared with conventional single-layer acquisitions performed at $100 \mathrm{kVp}$. As a compensation tube current-time product might be adapted to achieve equivalent $\mathrm{CTDI}_{\mathrm{vol}}$ to standard-, reduced-, or low-dose SLCT protocols. The results in this study show that patient radiation exposure can be restored to levels close to that of reduceddose SLCT with comparable image quality by adjusting automated exposure control (i.e., DRI). Image quality was comparable between $\mathrm{DLCT}_{\text {low }}$ and $\mathrm{SLCT}_{\text {low }}$ despite the changes in tube potential and the resulting changes in tube current-time product. Thus, a notable dose reduction due to adjusted automatic exposure control can be achieved in DLCT protocols compared to standard protocols, but further reductions might be possible by fine-tuning the lower threshold for tube current-time product.

Previously, Van Ommen et al [15] had already shown that DLCT can be used for a broad range of applications without increasing patient radiation exposure compared to normaldose SLCT. However, neither quantitative nor qualitative image quality was evaluated in this study. Another aspect of our

Table 5 Results of the quantitative image quality evaluation: CT numbers of evaluated organs and vessels in the thorax, upper abdomen, and lower abdomen. Statistical significance of results is indicated by italics

\begin{tabular}{|c|c|c|c|c|c|c|}
\hline \multirow{2}{*}{$\begin{array}{l}\text { Quantitative parameters } \\
\text { Anatomic location }\end{array}$} & \multirow[t]{2}{*}{$\operatorname{SLCT}_{\text {low }}(*)$} & \multirow[t]{2}{*}{$\operatorname{DLCT}_{\text {low }}(* *)$} & \multirow[t]{2}{*}{$\operatorname{DLCT}_{\text {high }}(* * *)$} & \multicolumn{3}{|l|}{$p$ value } \\
\hline & & & & $*$ vs. $* *$ & $*$ vs. $* * *$ & $* *$ vs. $* * *$ \\
\hline \multicolumn{7}{|l|}{ Thorax } \\
\hline Subcutaneous fat (HU) & $-115.7 \pm 12.0$ & $-110.5 \pm 12.9$ & $-113.0 \pm 12.1$ & 0.0013 & 0.035 & 0.23 \\
\hline Lung (HU) & $-875.5 \pm 39.8$ & $-877.1 \pm 43.6$ & $-877.1 \pm 49.2$ & 0.54 & 0.80 & 0.42 \\
\hline Trachea $(\mathrm{HU})$ & $-987.8 \pm 14.0$ & $-991.9 \pm 7.2$ & $-993.9 \pm 7.6$ & 0.0018 & 0.0002 & 0.58 \\
\hline \multicolumn{7}{|l|}{ Upper abdomen } \\
\hline Subcutaneous fat (HU) & $-114.4 \pm 10.4$ & $-104.7 \pm 14.3$ & $-106.6 \pm 16.2$ & $<0.0001$ & $<0.0001$ & 0.81 \\
\hline Liver (HU) & $113.0 \pm 18.7$ & $106.7 \pm 18.8$ & $99.3 \pm 22.3$ & $<0.0001$ & $<0.0001$ & 0.0015 \\
\hline Portal vein (HU) & $173.9 \pm 21.3$ & $151.5 \pm 20.3$ & $152.2 \pm 22.4$ & $<0.0001$ & $<0.0001$ & 0.27 \\
\hline Aorta (HU) & $161.9 \pm 23.2$ & $143.8 \pm 19.2$ & $142.7 \pm 22.6$ & $<0.0001$ & $<0.0001$ & 0.51 \\
\hline Spleen (HU) & $118.1 \pm 11.9$ & $107.3 \pm 11.7$ & $106.8 \pm 16.0$ & $<0.0001$ & $<0.0001$ & 0.62 \\
\hline \multicolumn{7}{|l|}{ Lower abdomen } \\
\hline Subcutaneous fat (HU) & $-118.0 \pm 8.7$ & $-109.4 \pm 10.2$ & $-111.5 \pm 10.4$ & $<0.0001$ & $<0.0001$ & 0.51 \\
\hline Psoas muscle (HU) & $59.3 \pm 8.2$ & $58.6 \pm 5.4$ & $59.5 \pm 6.0$ & 0.16 & 0.93 & 0.21 \\
\hline
\end{tabular}


Fig. 5 Results of the qualitative analysis showing the percentage of amount of received ratings, with best image quality observed for $\mathrm{DLCT}_{\text {high. }}$. Image quality for DLCT $_{\text {low }}$ was equal to SLCT $_{\text {low }}$

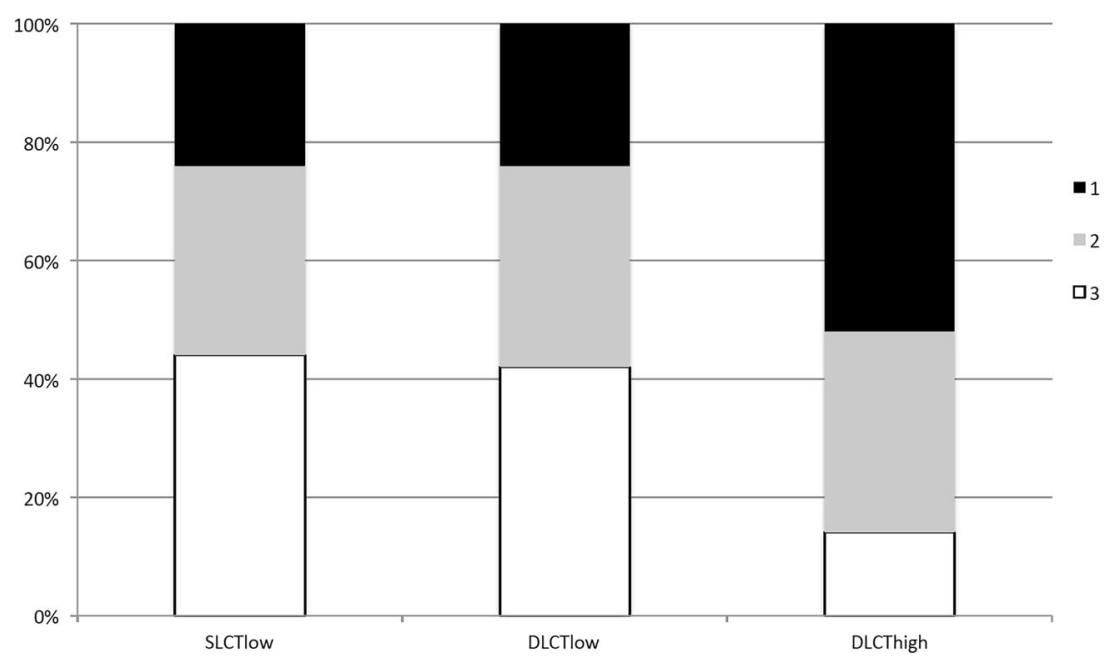

study was the adaptation of DLCT to match previous lowdose $\mathrm{SLCT}_{\text {low }}$ with $\mathrm{DLCT}_{\text {low }}$, which shows lower radiation doses than the current literature $[15,16]$. Moreover, the results show that even further dose reduction could be possible, especially in the thorax region where the automated exposure control reached the lower threshold of tube current-time product set in the acquisition protocol. According to the presented results, further dose reduction might be achieved by reducing the lower limit of the tube current from 65 to $55 \mathrm{mAs}$. According to Nagayama et al [17], DLCT showed a lower radiation dose than SLCT at equal tube potential of $120 \mathrm{kVp}$. However, with regard to image quality of conventional DLCT images, which is currently still the most frequently used reconstruction in clinical settings, no comparison was made with SLCT. Conventional CT images of dose equivalent DLCT and SLCT were already compared in a phantom study showing higher CNR but higher noise for DLCT compared to SLCT for $80-140 \mathrm{kVp}$, which was partly in agreement with the results presented here [18]. It should also be noted that despite matched CTDI ${ }_{\mathrm{vol}}$ between $\mathrm{SLCT}_{\text {low }}$ and $\mathrm{DLCT}_{\text {low }}$, as configured in the acquisition protocol (Table 1), the resulting $\mathrm{CTDI}_{\mathrm{vol}}$ for $\mathrm{DLCT}_{\text {low }}$ was higher than that for the $\mathrm{SLCT}_{\text {low }}$ (Table 2), which could be connected to the lower limit for the tube current mentioned above.

In general, the aspect of patient radiation exposure in spectral imaging has already been discussed in previous studies. No radiation dose increase is necessary for dual-source, dualenergy scans without compromises in image quality of the thorax and abdomen [19-21]. Rapid voltage switching dualenergy acquisition has shown ambiguous results. Singh et al showed dose equivalence to dual-source, dual-energy acquisition but with inferior image quality whereas other authors state that rapid voltage switching acquisition results in higher patient's radiation [22, 23].

One limitation of the study is that the patients included in the quantitative evaluation were not scanned with all acquisition protocols, as 6 out of 31 patients were only scanned with two of the three protocols. As the quantitative evaluation did not rely on pairwise comparisons, but used an unbalanced design instead, it was possible to include patients where one of the acquisitions was missing as well as to include multiple acquisitions of the same patient. This was done to increase the statistical power of the results, as the overall number of patients included in this study was relatively low, which is another limitation. Because of the limited number of patients, the transferability of the obtained results to clinical practice may be limited as well. One of the reasons for the limited number of patients in this study is the restriction to a single acquisition protocol, which, even though it is the most frequently used acquisition protocol at that CT scanner, limits the overall number of eligible patients and also limits the transferability of the obtained results to other acquisition protocols. However, comparing different protocols at once would have led to an unnecessary complexity of the evaluation, as additional variables would have to be considered. This is also true for the inclusion of arterial-phase imaging, which is more susceptible to changes in patient habitus, cardiac output, and underlying disease (e.g., differences in enhancement patterns for different tumors, as evident in pancreatic carcinoma [24]). While the results achieved in this study are specific for the acquisition protocol and body region, and obtained from a limited number of patients, and reductions in patient radiation exposure may be larger or smaller for other protocols or patient collectives, which has to be the subject of further evaluation in the future, we do expect that the results are nonetheless fairly generalizable, as we chose to evaluate a single, widely applicable acquisition protocol that makes up the bulk of acquisitions at the evaluated CT scanner. Even though there is no clear consensus on the definition of low dose, with an average patient dose of $60 \%$ below the applicable DRL, the evaluated acquisition protocol in this study qualifies as "reduced dose" [25]. 
Another limitation of this study and the DLCT acquisition protocols with increased tube potential might be the inferior conspicuity of vessel structures at higher tube potential, as mean photon energies closer to the k-edge of iodine provide improved image contrast [26]. However, with the additional possibility of spectral post-processing provided by DLCT, vessel delineation might be improved with virtual monochromatic images at lower virtual photon energies [27]. Furthermore, only conventional images of DLCT were analyzed in this study, even though spectral imaging was the main reason for the tube potential increase. Nonetheless, the number of potential spectral post-processing applications is very large and the quantification of the potential benefit from the additional spectral information for clinical routine is beyond the scope of this study.

Regarding the results, the inter-reader agreement for qualitative analysis was found to be moderate. However, this might be a consequence of the forced choice method used for evaluation combined with the low number of available choice (3 images). In consequence, a disagreement of ranking for one patient always consists of at least two further mismatches, resulting in a moderate Cohen's kappa. As all CT images were of diagnostic quality, the forced choice method was able to provide a side-by-side comparison, allowing to identify even small differences in image quality that might otherwise remain hidden with more conventional methods, like rating image quality on a Likert scale. Moreover, the quantitative analysis found differences in the delineation of vasculature as shown by the differences in $\mathrm{CT}$ numbers measured in the vessels. These differences did not lead to significant differences in subjective image quality, as delineation of vasculature is but one aspect of overall image quality. While Singh et al or Tabari et al $[22,28]$ suggest to link the evaluation of image quality to the clinical task of lesion detection, this approach was not applicable to this study as not all patients had lesions in all three evaluated body regions. In consequence, future studies may evaluate different aspects of image quality to provide a more complete evaluation for different clinical tasks.

\section{Conclusion}

Overall, image quality of $\mathrm{DLCT}_{\text {low }}$ was comparable to $\mathrm{SLCT}_{\text {low }}$ and a low-dose acquisition was possible using dual-layer technique, which has the additional benefit of providing spectral information. With $\mathrm{DLCT}_{\text {low }}, \mathrm{CTDI}_{\mathrm{vol}}$ could be reduced by $28 \%$ compared to $\mathrm{DLCT}_{\text {high }}$, but remained above that of $\mathrm{SLCT}_{\text {low. }}$. Further reduction of patient radiation exposure for the thorax seems possible.

Acknowledgements Dr. Thuy Duong Do was supported by a grant from the Medical Faculty of the University of Heidelberg.
Funding information Open Access funding provided by Projekt DEAL. This study has received funding by the Medical Faculty of the University of Heidelberg.

\section{Compliance with ethical standards}

Guarantor The scientific guarantor of this publication is Dr. Stephan Skornitzke.

Conflict of interest Stephan Skornitzke has ownership interests in investment funds containing stock of healthcare companies. Dr. Wolfram Stiller is a member of the CT Advisory Board of Philips Medical Systems. The authors of this manuscript declare no relationships with any companies whose products or services may be related to the subject matter of the article.

Statistics and biometry Dr. Stephan Skornitzke has significant statistical expertise.

Informed consent Written informed consent was waived by the Institutional Review Board.

Ethical approval Institutional Review Board approval was obtained.

Study subjects or cohorts overlap Study subjects or cohorts have not been previously reported.

Methodology

- Retrospective

- Experimental

- Performed at one institution

Open Access This article is licensed under a Creative Commons Attribution 4.0 International License, which permits use, sharing, adaptation, distribution and reproduction in any medium or format, as long as you give appropriate credit to the original author(s) and the source, provide a link to the Creative Commons licence, and indicate if changes were made. The images or other third party material in this article are included in the article's Creative Commons licence, unless indicated otherwise in a credit line to the material. If material is not included in the article's Creative Commons licence and your intended use is not permitted by statutory regulation or exceeds the permitted use, you will need to obtain permission directly from the copyright holder. To view a copy of this licence, visit http://creativecommons.org/licenses/by/4.0/.

\section{References}

1. Hickethier T, Byrtus J, Hauger M et al (2018) Utilization of virtual mono-energetic images (MonoE) derived from a dual-layer spectral detector CT (SDCT) for the assessment of abdominal arteries in venous contrast phase scans. Eur J Radiol 99:28-33

2. Doerner J, Luetkens JA, Iuga AI et al (2018) Poly-energetic and virtual mono-energetic images from a novel dual-layer spectral detector CT: optimization of window settings is crucial to improve subjective image quality in abdominal CT angiographies. Abdom Radiol (NY) 43:742-750

3. Doerner J, Hauger M, Hickethier T et al (2017) Image quality evaluation of dual-layer spectral detector CT of the chest and comparison with conventional CT imaging. Eur J Radiol 93:52-58 
4. Borggrefe J, Neuhaus VF, Le Blanc M et al (2019) Accuracy of iodine density thresholds for the separation of vertebral bone metastases from healthy-appearing trabecular bone in spectral detector computed tomography. Eur Radiol 29:3253-3261

5. Lennartz S, Laukamp KR, Neuhaus V et al (2018) Dual-layer detector CT of the head: initial experience in visualization of intracranial hemorrhage and hypodense brain lesions using virtual monoenergetic images. Eur J Radiol 108:177-183

6. Huda W, Scalzetti EM, Levin G (2000) Technique factors and image quality as functions of patient weight at abdominal CT. Radiology 217:430-435

7. Mettler FA Jr, Bhargavan M, Faulkner K et al (2009) Radiologic and nuclear medicine studies in the United States and worldwide: frequency, radiation dose, and comparison with other radiation sources-1950-2007. Radiology 253:520-531

8. Strahlenschutz Bf Röntgendiagnostik: Häufigkeit und Strahlenexposition. Available via https:/www.bfs.de/DE/themen/ ion/anwendung-medizin/diagnostik/roentgen/haeufigkeitexposition.html. Accessed 30 Nov 2019

9. Garcia-Garrigos E, Arenas-Jimenez JJ, Sanchez-Paya J (2018) Best protocol for combined contrast-enhanced thoracic and abdominal CT for lung cancer: a single-institution randomized crossover clinical trial. AJR Am J Roentgenol 210:1226-1234

10. Pahn G, Skornitzke S, Schlemmer HP, Kauczor HU, Stiller W (2016) Toward standardized quantitative image quality (IQ) assessment in computed tomography (CT): a comprehensive framework for automated and comparative IQ analysis based on ICRU report 87. Phys Med 32:104-115

11. Gur D, Rubin DA, Kart BH et al (1997) Forced choice and ordinal discrete rating assessment of image quality: a comparison. J Digit Imaging 10:103-107

12. American Association of Physicists in Medicine (AAPM) Task Group 204 (2011): Size-specific dose estimates (SSDE) in pediatric and adult body CT examinations. Available via https://www.aapm. org/pubs/reports/RPT_204.pdf. Accessed 20 Dec 2019

13. Brink JA, Morin RL (2012) Size-specific dose estimation for CT: how should it be used and what does it mean? Radiology 265:666668

14. Landis JR, Koch GG (1977) The measurement of observer agreement for categorical data. Biometrics 33:159-174

15. van Ommen F, de Jong H, Dankbaar JW, Bennink E, Leiner T, Schilham AMR (2019) Dose of CT protocols acquired in clinical routine using a dual-layer detector CT scanner: a preliminary report. Eur J Radiol 112:65-71

16. Haneder S, Siedek F, Doerner J et al (2018) Thoracic-abdominal imaging with a novel dual-layer spectral detector CT: intra- individual comparison of image quality and radiation dose with 128-row single-energy acquisition. Acta Radiol 59:1458-1465

17. Nagayama Y, Nakaura T, Oda S et al (2018) Dual-layer detector CT of chest, abdomen, and pelvis with a one-third iodine dose: image quality, radiation dose, and optimal monoenergetic settings. Clin Radiol 73:1058 e1021-1058 e1029

18. van Ommen F, Bennink E, Vlassenbroek A et al (2018) Image quality of conventional images of dual-layer SPECTRAL CT: a phantom study. Med Phys 45:3031-3042

19. Schenzle JC, Sommer WH, Neumaier K et al (2010) Dual energy CT of the chest: how about the dose? Invest Radiol 45:347-353

20. Uhrig M, Simons D, Kachelriess M, Pisana F, Kuchenbecker S, Schlemmer HP (2016) Advanced abdominal imaging with dual energy $\mathrm{CT}$ is feasible without increasing radiation dose. Cancer Imaging 16:15

21. Siegel MJ, Curtis WA, Ramirez-Giraldo JC (2016) Effects of dualenergy technique on radiation exposure and image quality in pediatric body CT. AJR Am J Roentgenol 207:826-835

22. Singh R, Sharma A, McDermott S et al (2019) Comparison of image quality and radiation doses between rapid $\mathrm{kV}$-switching and dual-source DECT techniques in the chest. Eur J Radiol 119: 108639

23. Johnson TR (2012) Dual-energy CT: general principles. AJR Am J Roentgenol 199:S3-S8

24. Boland GW, O'Malley ME, Saez M, Fernandez-del-Castillo C, Warshaw AL, Mueller PR (1999) Pancreatic-phase versus portal vein-phase helical CT of the pancreas: optimal temporal window for evaluation of pancreatic adenocarcinoma. AJR Am J Roentgenol 172:605-608

25. Bankier AA, Kressel HY (2012) Through the looking glass revisited: the need for more meaning and less drama in the reporting of dose and dose reduction in CT. Radiology 265:4-8

26. Alkadhi H, Schindera ST (2011) State of the art low-dose CT angiography of the body. Eur J Radiol 80:36-40

27. Zopfs D, Lennartz S, Laukamp K et al (2018) Improved depiction of atherosclerotic carotid artery stenosis in virtual monoenergetic reconstructions of venous phase dual-layer computed tomography in comparison to polyenergetic reconstructions. Eur J Radiol 100: 36-42

28. Tabari A, Ramandeep S, Khera RD et al (2019) Can fully iterative reconstruction technique enable routine abdominal CT at less than 1 mSv? Eur J Radiol Open 6:225-230

Publisher's note Springer Nature remains neutral with regard to jurisdictional claims in published maps and institutional affiliations. 